\title{
Basic structural and functional characteristics of the epidermal barrier in wild mammals living in different habitats and climates
}

\author{
Wilfried Meyer • Judith Schmidt • Johannes Kacza • \\ Roger Busche • Hassan Y. Naim • Ralf Jacob
}

Received: 4 November 2010 /Revised: 13 January 2011 / Accepted: 14 January 2011 /Published online: 29 January 2011

(C) Springer-Verlag 2011

\begin{abstract}
Based on the combination of standard light and transmission electron microscopy, cryo-SEM, immunohistochemistry and a new sensitive glycolipid histochemical technique (5-hexadecanoylaminofluorescein staining, laser scanning microscopy), including densitometrical evaluation, our approach gives for the first time an overview of the specific biology of the epidermal permeability barrier in wild mammals (20 species from five orders), living under varying (aquatic or moist to dry) habitat conditions. The results obtained emphasised that the barrier region in most of the species studied is a continuous zone (thickness, 0.1 and $3 \mu \mathrm{m}$ ) between the upper cells of the stratum granulosum and the inner cells of the stratum corneum conjunctum, normally present as a homogeneous glycolipid layer originating from fusion of lamellar body contents after
\end{abstract}

Communicated by C. Gortázar

W. Meyer $(\bowtie) \cdot J$. Schmidt

Institute for Anatomy,

University of Veterinary Medicine Hannover, Foundation,

Bischofsholer Damm 15,

30173 Hannover, Germany

e-mail: wilfried.meyer@tiho-hannover.de

J. Kacza

Institute of Veterinary Anatomy, University of Leipzig,

04103 Leipzig, Germany

R. Busche $\cdot$ H. Y. Naim

Institute for Physiological Chemistry,

University of Veterinary Medicine Hannover, Foundation,

30559 Hannover, Germany

R. Jacob

Institute of Cytobiology and Cytopathology,

University of Marburg,

35033 Marburg, Germany exocytotic activities of the granular cells. However, this finding did not apply to all of the species studied, i.e., the Wild boar, the Common seal and the three large species with a very thick vital epidermis, the African elephant, the hippopotamus and the common dolphin, exhibited variations from the basic scheme. Densitometric evaluation of the 5-hexadecanoylaminofluorescein staining revealed that reaction intensity was not only generally related to the habitat conditions but also to vital epidermis thickness and hair density. The immunohistochemical demonstration of $\mathrm{Na}^{+} / \mathrm{H}^{+}$exchanger 1 corroborated for all wild mammals studied that this important regulator of $\mathrm{pH}$ conditions during barrier formation is continuously produced in the epidermis. The variations in barrier biology observed for some species obviously had to be developed in relation to animal size (or body size area) and hair coat density, but, particularly, by the specific adaptation of certain mammalian groups to the aquatic environment. In the latter case, the typical barrier zone system was lost, as in the hippopotamus or the cetaceans.

Keywords Barrier Epidermis · Wild mammals · Biotope adaptation $\cdot$ Glycolipids

\section{Introduction}

The integument represents an effective barrier between the organism and the environment, preventing invasion of pathogens and fending off chemical and physical assaults, as well as the unregulated loss of water and solutes. The mammalian integument, in particular, mediates this broad spectrum of protective functions, whereby permeability and antimicrobial abilities are both co-regulated and interdependent, overlapping through the dual activities of their 
lipid/protein constituents (Elias 2007; Heung et al. 2006; Aberg et al. 2008).

As a very important functional feature during epidermal differentiation, glycolipids are continuously synthesised in the keratinocytes and extruded from the cells of the stratum granulosum as lamellar bodies (membrane coating granules) into extracellular domains. Here, they form a thin but quite stable extracellular lipid-enriched layer, the epidermal permeability barrier, between the stratum granulosum and the stratum corneum conjunctum (see e.g. Elias et al. 1977; Meyer 1986; Goldsmith 1991; Meyer et al. 2009). The glycolipids in question are ceramides (glycosphingolipids), whereby in the barrier region, glucosylceramides are most important (Holleran et al. 1993, 2006; Hamanaka et al. 2005; Jennemann et al. 2007; Mizutani et al. 2009). In the latter case, the formation of lamellar bodies, in particular, prevents uncontrolled water loss through the skin as a basic critical feature of the evolution of terrestrial vertebrates to survive within the new biotopes on land. This means, for example, that fundamental mechanisms for adjusting the skin water barrier comprise changes in barrier thickness, composition and physicochemical properties of cutaneous lipids and/or geometry of the barrier within the epidermis (Lillywhite 2006).

Regarding the successful adaptation of modern mammalian groups to more or less dry terrestrial habitats and also considering a specific secondary adaptation to the aquatic medium, as known for example from pinnipeds or cetaceans, our study includes a comparative approach to demonstrate the typical structure and function of the epidermal permeability barrier of wild mammals related to their general biotope types. Regarding this idea, we took into account, additionally, the study of specific light and electron microscopical features, including the thickness of the vital epidermis or hair coat density.

Considering the application of relevant methods to show typical functional aspects, we had to realise, first of all, that lipids, or better lipoids, are a chemically very heterogeneous group of substances, all of which are insoluble in water but soluble in a variety of non-polar organic solvents. As a result, the effectivity of different light microscopical, electron microscopical and histochemical methods was subject to certain limitations (for literature, see e.g. Bayliss High 1984; Pearse 1985; Meyer et al. 2009). Nevertheless, it was possible to use routine methods in combination with several modern techniques, such as cryo-SEM that ensures an almost lifelike view by means of careful and artifact-free tissue processing (Meyer et al. 2007) and specific plastic resin embedding and staining to the skin material we had to our disposal. We could also use a new technique that guaranteed practically no tissue shrinkage and, as combined with confocal laser scanning microscopy (LSM), a structurally reliable demonstration of glycolipids by a very sensitive fluorescence dye (Meyer et al. 2009). For an indirect control of barrier formation, the $\mathrm{Na}^{+} / \mathrm{H}^{+}$exchanger 1 (NHE-1) was demonstrated immunohistochemically. The members of this group of plasma membrane intrinsic proteins are expressed ubiquitously in all tissues, activated by numerous growth factors and implicated in $\mathrm{pH}$ homeostasis, cell volume regulation and proliferation, or the adjustment of intracellular pH (e.g. Hachem et al. 2003; Slepkow et al. 2007; Koliakos et al. 2008). In the keratinocytes, NHE-1 is essential for acidifying the lipid microdomains (lipid rafts; Wenneckes et al. 2009) and very important for barrier repair or recovery (e.g. Behne et al. 2002; Hachem et al. 2005).

In view of a successful application of all techniques, our approach gives for the first time an overview of the specific biology of the epidermal permeability barrier in wild mammals from different systematic groups, living under varying habitat or climate conditions. Moreover, in this context, we discuss the problem of a correct definition of the epidermal barrier region that, unfortunately, still today is only based on results from humans or laboratory animals and not on ecologically relevant information of mammalian skin biology.

\section{Materials and methods}

Animals and sampling

Skin material was collected from the dorsolateral body region of 20 adult wild mammalian species originating from four more or less dry or humid biotope groups (Table 1) and additionally from four representatives of typical domesticated mammals with a very dense, dense or sparse hair coat: laboratory rat (Rattus norvegicus f. dom., 4 우오, Fischer 344, Lewis), cat (mixed breed, Felis silvestris f. dom., 2 우), cattle (Holstein Friesian, 2 우, Bos primigenius f. dom.) and pig (German landrace, Sus scrofa f. dom., 4 우). The latter animals were used to obtain fresh material immediately stored in liquid nitrogen for an epidermal analysis using cryo-SEM; such an approach was only rarely possible with the skin material from the wild mammals.

The wild animals used in the course of this study were kept and/or legally euthanised in the Wisent Zoo (Springe, Germany), the Zoo Krefeld (Krefeld, Germany), the Senckenberg Museum of Natural History Goerlitz (Goerlitz, Germany) or the respective university institutions (Institute of Zoology, Institute of Anatomy, Institute of Pharmacology, Toxicology and Pharmacy and the Small Animal Clinic, all of the University of Veterinary Medicine Hannover Foundation, Hannover, Germany; Center for Morphology, Dr. Senckenberg Anatomy, University of Frankfurt/M.; Department of Anatomy of Animals, University of Life Sciences, Poznan, Poland) or 
Table 1 Mammalian species studied and related climates

\begin{tabular}{|c|c|}
\hline Biotope & Number and sex \\
\hline \multicolumn{2}{|l|}{ Aquatic (variable marine climate) } \\
\hline Common dolphin (Delphinus delphis) & 3 우, $1 \hat{\jmath}$ \\
\hline \multicolumn{2}{|l|}{ Semiaquatic (arctic, temp., or trop. climate) } \\
\hline Nutria (Myocastor coypus) & 3 우, 1 juv. \\
\hline Beaver (Castor fiber) & 3 우우 \\
\hline Capybara (Hydrochoeris hydrochaeris) & 2 우, $1 \hat{\jmath}$ \\
\hline Otter (Lutra lutra) & 3 우, $2 \hat{\jmath}$ \\
\hline Polar bear (Ursus maritimus) & 2 웅, $1 \hat{\jmath}$ \\
\hline Common seal (Phoca vitulina) & $5 \uparrow, 1 \hat{\jmath}$ \\
\hline Hippopotamus (Hippopotamus amphibius) & 1 , $1 \hat{\jmath}, 1$ juv. \\
\hline \multicolumn{2}{|l|}{ Generally moist (tropical climate) } \\
\hline Jaguar (Panthera onca) & $1 \lesssim$ \\
\hline \multicolumn{2}{|l|}{ Variably moist or dry (temperate climate) } \\
\hline Brown rat (Rattus norvegicus) & 5 우, $2 \hat{\jmath}$ \\
\hline Wolf (Canis lupus) & $3 \uparrow+, 2 \hat{\jmath}$ \\
\hline Brown bear (Ursus arctos) & 2 우, 1 ๙ \\
\hline Wild boar (Sus scrofa) & 6 우, $2 \hat{\jmath}$ \\
\hline Wild cat (Felis silvestris) & 2 ๆํ, 2 ठठ \\
\hline \multicolumn{2}{|l|}{ Generally dry (tropical climate) } \\
\hline African lion (Panthera leo) & $3 ㅇ ㅜ ㅇ ㅜ$ \\
\hline African buffalo (Syncerus caffer) & $2 \nmid 90$ \\
\hline Kongoni (Alcelaphus buselaphus) & 2 웅, $1 \hat{\jmath}$ \\
\hline Oryx (Oryx gazella) & 2 qㅇ, $1 \hat{\jmath}, 1$ juv. \\
\hline Greater kudu (Tragelaphus strepsiceros) & 2 우, $1 \hat{\jmath}$ \\
\hline African elephant (Loxodonta africana) & $3+9,1 \hat{\jmath}$ \\
\hline
\end{tabular}

legally hunted in Germany (Veterinary Investigation Centre for Fish and Fish Products, Cuxhaven, Germany) and in Africa (Institute of Wildlife Research, University of Veterinary Medicine Hannover Foundation, Hannover, Germany).

\section{Fixation}

Samples of about $1 \mathrm{~cm}^{3}$ were directly immersed in Bouin's solution for $48 \mathrm{~h}$, washed several times in 70\% ethanol containing several drops of ammonia (concentration) and stored in 80\% ethanol (Boeck 1989). Bouin's fluid was chosen because it is less damaging to normal tissue structure than formaldehyde alone and to avoid tissue shrinkage already during the fixation step (Pearse 1985). It was also used because a more exact substance localisation can be obtained for immunohistochemical purposes. Additionally, other skin samples were fixed in $4 \%$ calcium acetate formalin after Lillie (Lillie and Fullmer 1976); after fixation, this material was rinsed and stored in $70 \%$ ethanol. Formalin was used to obtain a good fixation of compound lipids (Lillie and Fullmer 1976; for review of important features of fixation, see Hornickel et al. 2011; Meyer and Hornickel 2011).

Independent of the storage in liquid nitrogen directly after sampling, the freshly obtained skin material of the four domesticated mammals was fixated in the two media referred to beforehand, and small skin blocks of the Wolf, the Wild boar and the Wild cat were also immersed in Karnovsky's fluid (Karnovsky 1965) for TEM analysis.

\section{Embedding and cutting}

The samples fixed in Bouin's solution and Ca-formol were embedded in paraffin wax (Paraplast plus, Covidien, Neustadt, Germany) according to standard protocols (Boeck 1989). The paraffin blocks were sectioned with a rotary microtome (Reichert-Jung, Nussloch, Germany), and sections not thicker than $5 \mu \mathrm{m}$ were used for the immunohistochemical experiments conducted.

Moreover, for histological structure control and glycolipid staining, small tissue blocks of about $0.5 \mathrm{~mm}^{3}$ were prepared, carefully dehydrated with graded ethanol (70, 80, 90, $2 \times 96$, $2 \times 100 \%$ ) and embedded in the water-soluble and rather shrinkage-free 2-hydroxy-methacrylate Technovit ${ }^{\circledR} 7100$ (Heraeus-Kulzer, Wehrheim, Germany; Gerrits and Smid 1983; Hanstede and Gerrits 1983). Three-micrometre plastic sections were cut with a motor-driven rotation microtome (model 1140, Autocut, Reichert-Jung) and transferred to slides. In view of the fact that embedding without previous dehydration, using aqua dest. as dissolution medium for the plastic resin, had not produced differences in reaction intensity compared to embedding via ethanol (Meyer et al. 2009), this step was abandoned.

\section{Standard TEM}

The Karnovsky-fixed TEM material was washed several times in PBS and postfixed in buffered $1 \%$ osmium tetroxide (Millonig 1961). Additionally, several small skin blocks from wild mammals fixed in Bouin's fluid or formalin were postfixed in osmium tetroxide and handled together with the samples fixated in the Karnovsky's fluid, i.e. after careful dehydration in graded ethanol, all samples were embedded in Epon 812 (Serva Electrophoresis, Heidelberg, Germany; Luft 1961) and cut with a diamond knife on the ultramicrotome Ultracut E (Leica Microsystems, Wetzlar, Germany). Semithin sections were stained with $0.2 \%$ toluidine blue $\mathrm{O}$ (Richardson et al. 1960); thin sections ( $<100 \mathrm{~nm})$ were contrasted with methanolic uranyl acetate (Stempak and Ward 1964) and lead citrate (Reynolds 1963) and viewed in the electron microscopes EM10 and EM10C (Carl Zeiss, Jena, Germany) operated at $60 \mathrm{kV}$. 


\section{Cryo-SEM}

Thin skin pieces $(2 \times 4 \times 8 \mathrm{~mm})$ were directly immersed in liquid nitrogen $\left(\mathrm{LN}_{2}\right)$ and stored separately therein until use. For analysis, a sample was transferred to a cryo-sample loading device (Bal-Tec AG, Balzers, Liechtenstein) and carefully mounted in a specially designed clamping device on top of an object table. A $\mathrm{LN}_{2}$-cooled vacuum cryotransfer system (cryo-shuttle VCT 100, Bal-Tec), in which the sample was kept in an atmosphere of evaporising nitrogen for about $15 \mathrm{~s}$, was used to transfer the object table in a freeze-etch/freeze-fracture unit (MED 020, Bal-Tec). Therein, it was placed on a cryo stage, where it was kept under high vacuum $\left(5 \times 10^{-6} \mathrm{mbar}\right)$ at a minimum temperature of $-150^{\circ} \mathrm{C}$. Then, the skin sample was hit once with a blade, thus creating a freeze fracture from the outermost to the deeper skin layers. Subsequently, the surface of the sample was sputter-coated twice with gold palladium from opposite directions (40 s, $40 \mathrm{~mA}, 5 \times 10^{-2} \mathrm{mbar}$, argon). Using the shuttle, the coated sample was transferred onto the cryo-stage (Bal-Tec) of an adapted LEO $1430 \mathrm{vp}$ scanning electron microscope (Zeiss) and inspected at minimum vacuum of $9 \times 10^{-6}$ mbar with an accelerating voltage of $10 \mathrm{kV}$.

\section{LM staining, epidermal thickness and hair density}

In order to evaluate the quality of tissue preservation and for general structural analysis, paraffin and plastic sections of all collected samples were stained with haematoxylin and eosin (H\&E, haematoxylin according to Delafield; Boeck 1989). Only H\&E-stained sections of samples that had been embedded in water-soluble plastic resin were used for thickness analysis of the vital epidermis. Between 80 and 120 measurements were recorded for each of the wild species, and mean values and standard deviations were evaluated with the help of the computer-assisted CUE 3 system (Olympus, Image Analysis, version 4.5, 1993). Unless adopted from the cited literature (see Table 2), the number of hair follicles per square centimetre of the dorsal body region was determined from horizontal sections using a Zeiss microscope equipped with a drawing tube. For each individual, the primary and secondary hairs were counted on three-section areas of $2 \times 2 \mathrm{~mm}$. The results were used to evaluate the number of hair follicles per square centimetre and the percentage of wool hairs, and mean values including standard deviations were calculated.

\section{Glycolipid staining}

For glycolipids, the plastic sections were stained with the green fluorescence dye 5-hexadecanoylaminofluorescein (HEDAF; Molecular Probes Inc., Eugene, OR, USA) according to Meyer et al. (2009). The dye particularly binds to polar membrane-associated glycolipids and consists of two parts, a fluorescein molecule and an attached palmitoyl chain. All sections were viewed on a Leica TCS SP2 confocal laser scanning microscope (LSM; $488 \mathrm{~nm}$ excitation, $519 \mathrm{~nm}$ emission). For a control of the lipid staining, the material was treated in two different ways: (a) plastic sections were immersed at room temperature for 48 and $72 \mathrm{~h}$ in acetone (abs.) prior to staining with the two dyes; (b) small skin samples were immersed in a mixture of chloroform (abs.) and methanol (abs.) for 24, 36 and $48 \mathrm{~h}$ at room temperature, afterwards embedded via ethanol (abs.) in Technovit ${ }^{\circledR} 7100$ and the sections were treated as described beforehand to achieve glycolipid staining.

\section{LSM measurements}

Confocal images (area size $10 \times 10 \mu \mathrm{m}$ ) of plastic sections were acquired on a Leica TCS SP2 microscope using a 40× oil planapochromate lens (Leica Microsystems, Wetzlar, Germany) with a 488-nm argon laser excitation essentially as described before (see, e.g. Delacour et al. 2008). Fluorescence of HEDAF inserted into lipid membranes was recorded by emission detection in an interval between 519 and $676 \mathrm{~nm}$. Thereby, the photomultiplier sensitivity was kept at a quite constant level. Thirty to 50 fluorescent tissue zones of the upper epidermis with the barrier region were quantified for each species. For every image, about 20 areas in the barrier zone or the underlying vital epidermis were confined as region of interest (ROI). The intensity and the area of each ROI (square nanometre) were collected with the Leica LCS software package and exported into an excel sheet. Intensity values per square nanometre were evaluated, and mean values including standard deviations were calculated for each species. The results of the measurements were evaluated statistically for significant differences between each species by an analysis of variance in a split plot design combined with a $t$ test for paired data.

\section{Immunohistochemical staining}

Five-micrometre paraffin sections were deparaffinised in Histoclear (Life Science Int.) and carefully hydrated through descending concentrations of ethanol. Afterwards, these sections were stained for the determination of the $\mathrm{Na}^{+} / \mathrm{H}^{+}$exchanger 1 (NHE-1; dilution 1:50, 1:100; antimouse/rat, from rabbit, polyclonal; Biologo, Kronshagen, Germany). Following incubation overnight at $4^{\circ} \mathrm{C}$, the reaction was detected by a fluorescein isothiocyanate (FITC)-linked IgG system (Vector Labs., Braunschweig, Germany). One part of the sections was also digested for 30-60 min with $0.1 \%$ trypsin (from porcine pancreas, type II, crude; Sigma-Aldrich Chemie, Taufkirchen/Munich, 
Table 2 Hair density and epidermal thickness of the species studied

\begin{tabular}{|c|c|c|c|}
\hline Biotope & Hair density (dorsum) hairs $/ \mathrm{cm}^{2}$ & Wool hairs $\left(\%\right.$ of total hairs $\left./ \mathrm{cm}^{2}\right)$ & $\begin{array}{l}\text { Thickness vital epidermis } \\
\text { (dorsum) }(\mu \mathrm{m})\end{array}$ \\
\hline \multicolumn{4}{|l|}{ Aquatic (variable marine climate) } \\
\hline Common dolphin (Delphinus delphis) & Not present & & $1,516.67( \pm 51.64)$ \\
\hline \multicolumn{4}{|l|}{ Semiaquatic (arctic, temp., trop. climate) } \\
\hline Nutria (Myocastor coypus). & $14,250( \pm 4,300)$ & $92.3( \pm 5.53)$ & $26.78( \pm 4.23)$ \\
\hline Beaver (Castor fiber) & $32,430( \pm 5,540)$ & $95.6( \pm 4.50)$ & $9.94( \pm 2.46)$ \\
\hline Capybara (Hydrochoeris hydrochaeris) & $1,300( \pm 350)$ & $8.5( \pm 2.58)$ & $57.14( \pm 9.95)$ \\
\hline Otter (Lutra lutra) & $69,900( \pm 12,100)$ & $98.74(8.92)$ & $8.69( \pm 2.49)$ \\
\hline Polar bear (Ursus maritimus) & $2,900( \pm 1,220)$ & $66.0( \pm 22.60)$ & $22.13( \pm 5.14)$ \\
\hline Common seal (Phoca vitulina) & $1,240( \pm 170)$ & $72.5( \pm 2.50)$ & $43.85( \pm 8.36)$ \\
\hline Hippopotamus (Hippopotamus amphibius) & $110( \pm 40)$ & 0 & $158.90( \pm 25.14)$ \\
\hline \multicolumn{4}{|l|}{ Generally moist (tropical climate) } \\
\hline Jaguar (Panthera onca) & $2,900( \pm 1,300)$ & $76.3( \pm 12.40)$ & $17.53( \pm 3.03)$ \\
\hline \multicolumn{4}{|l|}{ Variably moist or dry (temp. climate) } \\
\hline Brown rat (Rattus norvegicus) & $13,940( \pm 2,100)$ & $75.5( \pm 20.90)$ & $10.74( \pm 3.64)$ \\
\hline Wolf (Canis lupus) & $5,490( \pm 620)$ & $82.9( \pm 3.40)$ & $17.43( \pm 3.18)$ \\
\hline Brown bear (Ursus arctos) & $2,570( \pm 1,220)$ & $65.0( \pm 18.24)$ & $23.56( \pm 3.86)$ \\
\hline Wild boar (Sus scrofa) & $240( \pm 25)$ & $75,5( \pm 16.60)$ & $20.75( \pm 5.97)$ \\
\hline Wild cat (Felis silvestris) & $12,800( \pm 4,200)$ & $91.7( \pm 2.47)$ & $12.60( \pm 3.65)$ \\
\hline \multicolumn{4}{|l|}{ Generally dry (tropical climate) } \\
\hline Lion (Panthera leo) & $1,880( \pm 850)$ & $75.3( \pm 13.41)$ & $21.17( \pm 4.11)$ \\
\hline African buffalo (Syncerus caffer) & $980( \pm 290)$ & $34.0( \pm 9.20)$ & $70.50( \pm 12.17)$ \\
\hline Kongoni (Alcelaphus buselaphus) & $2,310( \pm 350)$ & $75.0( \pm 6.92)$ & $28.25( \pm 4.06)$ \\
\hline Oryx (Oryx gazella) & $2,820( \pm 380)$ & $76.2( \pm 8.35)$ & $35.90( \pm 7.05)$ \\
\hline Greater kudu (Tragelaphus strepsiceros) & $1,800( \pm 260)$ & $72.8( \pm 5.80)$ & $42.75( \pm 10.82)$ \\
\hline African elephant (Loxodonta africana) & $20( \pm 15)$ & 0 & $104.64( \pm 25.49)$ \\
\hline
\end{tabular}

Hair density and thickness of the vital epidermis (all measurements after embedding in water soluble and shinkage free plastic resin) according to Meyer et al. (1981), Uhr (1984), Meyer (1986, 2010), Meyer (1996-2008, unpublished results), Bollhorn (1999), Kuhn (2009); the juvenile hippopotamus was not measured

Germany; Hautzer et al. 1980) or incubated for $30 \mathrm{~min}$ in TEC buffer at $90^{\circ} \mathrm{C}$ prior to the reaction. Control sections were incubated without the antibody and/or the visualisation system. The microscopic results were documented with a Zeiss Axioskop equipped with an epifluorescense device (FITC filter combination, BP450-490, FT510, LP520) and a digital camera (Olympus DP70). The software Olympus DP-SOFT (version 3.1 and 3.2) was applied for picture analysis.

\section{Results}

Light microscopical analysis

The thickness measurements of the vital epidermis of the dorsolateral body region revealed a large and diverging thickness spectrum comparing the different mammalian groups studied (Table 2), i.e. a very thin vital epidermis with $9-10 \mu \mathrm{m}$ in the beaver or the otter, and an extremely thick vital epidermis in the hairless common dolphin with about $1,500 \mu \mathrm{m}$. Among the terrestrial species, relatively thick values were only obtained with $100-160 \mu \mathrm{m}$ in the more or less hairless species, both the African elephant and the Hippopotamus, and to some extent in the African buffalo with about $70 \mu \mathrm{m}$. All the other species showed thicknesses between 10 and $50 \mu \mathrm{m}$; nevertheless, the relatively small and very sparsely haired semiaquatic capybara had astonishing values with about $60-70 \mu \mathrm{m}$.

When these results were submitted to regression analysis, it became obvious that only the medium hair density of species (see Table 2) living in variably moist or dry temperate and tropical climates $(r=0.83)$ or in the generally dry tropical climate $(r=0.81)$ was correlated with vital epidermis thickness (in both cases $p<0.001$ ). However, the missing correlation for the semiaquatic species living in artic, temperate or tropical climates was mainly due to the partly extremely high hair densities in the beaver and the otter. 
Independent of these measurements, histological analysis of the plastic sections, in particular, corroborated the view that even comparatively thin epidermis variations could be stained for specific lipid histochemical purposes (HEDAF) and analysed by LSM.

\section{TEM observations}

The electron microscopical results obtained emphasised a structurally always very clearly defined border zone between the upper cells of the stratum granulosum presenting a light cytoplasm, and the inner cells of the stratum corneum conjunctum, which were filled with condensed keratin filaments, with the exception of the Wild boar, and, particularly, the species with a very thick vital epidermis, the African elephant, the hippopotamus and the common dolphin. The intercellular space system between the two layers generally had a varying thickness between 0.1 and $1 \mu \mathrm{m}$ (Fig. 1), without, however, showing statistically relevant differences when the different mammalian groups studied were compared. Nevertheless, rather wide intercellular spaces $(0.5-1 \mu \mathrm{m})$ were visible in the thick epidermis of the capybara (Fig. 1a) and especially in that one of the large bovines studied (1-3.5 $\mu \mathrm{m}$; African buffalo, Kongoni, Oryx, Greater kudu), or the African lion (1-2.5 $\mu \mathrm{m})$, whereas most of the other terrestrial species had thinner spaces between both epidermal layers. The intercellular spaces contained different electron dense and electron light substances that were emptied into these spaces by exocytosis. Moreover, the stratum granulosum cells in most cases revealed varying amounts of cytoplasmic vesicles, including lamellar bodies (Fig. 1b), but just as often homogeneously stained electron-dense material (Fig. 1c). The maculae adhaerentes connecting the cells of both layers were numerous and normally had a length of about $1 \mu \mathrm{m}$, independent of the species analysed.

Clearly different observations were made regarding the epidermis of the African elephant, the hippopotamus and the common dolphin. In these species, but more distinctly visible in the dolphin, a differentiation between the stratum granulosum and the stratum corneum compactum was very difficult because a clear and separating intercellular space system or barrier region was missing. As general features, the species living in semiaquatic or aquatic milieus contained, besides varying amounts of lamellar bodies, always relatively high numbers of lipid vesicles in the cells of the two strata, and exhibited only very thin intercellular spaces of the same size, which became even more narrow in the stratum corneum conjunctum. Additionally, high numbers of desmosomal contacts could be identified, particularly in the dolphin. Basic features of the structural peculiarities described above could be detected also by light microscopy.

\section{Cryo-SEM observations}

The advantages of cryo-SEM that are primarily characterised by the demonstration of rather lifelike structures revealed the barrier system as a generally homogeneous layer of substances that originated from the fusion of vesicle contents after exocytotic activities of the cells of the stratum granulosum (Fig. 2a). The domesticated mammals that had to be examined indicated varying production activities of the four species studied. In the densely haired laboratory rat, a mulitude of fusing vesicle contents could be recognised (Fig. 2b), whereas in the sparsely haired domesticated pig, the number of vesicles was clearly smaller (Fig. 2c). Such observations are corroborated to a certain extent by the results of the measurements of the HEDAF staining intensities (Fig. 5).

\section{HEDAF glycolipid staining}

The HEDAF staining emphasised very distinctly by an intense light green reaction the barrier region between the stratum granulosum and the stratum corneum conjunctum in all wild mammalian species studied, with the exception of the Wild boar and the common seal, and again the common dolphin, the hippopotamus and the African elephant (see below). The positive staining was, in most cases, clearly limited to the barrier proper that appeared as a continuous layer (Fig. 3a-c), thus confirming the accumulation of glycolipids (glucosylceramides) produced by the granulosum cells and secreted from lamellar bodies into the space between the two epidermal layers in question. In the wild boar, a clear layer system of glycolipids was missing; instead, a patchy distribution of these substances between the lower lamellae of the stratum corneum was visible that sometimes also comprised the entire corneal layer system (Fig. 4a). The same pattern of glycolipid distribution was present in the seal (Fig. 4b), however, with clearly smaller glycolipid amounts or weaker reaction intensities, respectively. Independent of these findings, the intercellular substances of the other epidermal layers of most of the species studied normally showed only rather weak staining reactions, although in the large bovines (e.g. Greater kudu; Fig. 3d), these reactions often were more intense. In the species with very thick vital epidermis (dolphin, hippopotamus and elephant) where no definite barrier region could be detected, the staining was generally restricted to the intercellular spaces of the stratum spinosum and the stratum granulosum, whereas the stratum basale reacted negatively in this respect (Fig. 3c, elephant). In most epidermal sections of these large species partly living in different climates and habitats, also smaller or larger cytoplasmic vesicles of the upper cell layers exhibited a weak to medium green fluorescence, such as in the dolphin. 
Fig. 1 TEM results of barrier structure and production: a capybara, basic structure of the intercellular space system (asterisk), Bouin fixation; b wild boar, exocytosis of glycolipids by a lamellar body (arrow), Karnovsky fixation; c wild cat, exocytotic activities of cytoplasmic vesicles filled with electron-dense material (arrows), Karnovsky fixation; $S G C$ stratum granulosum, $S C C$ stratum corneum conjunctum

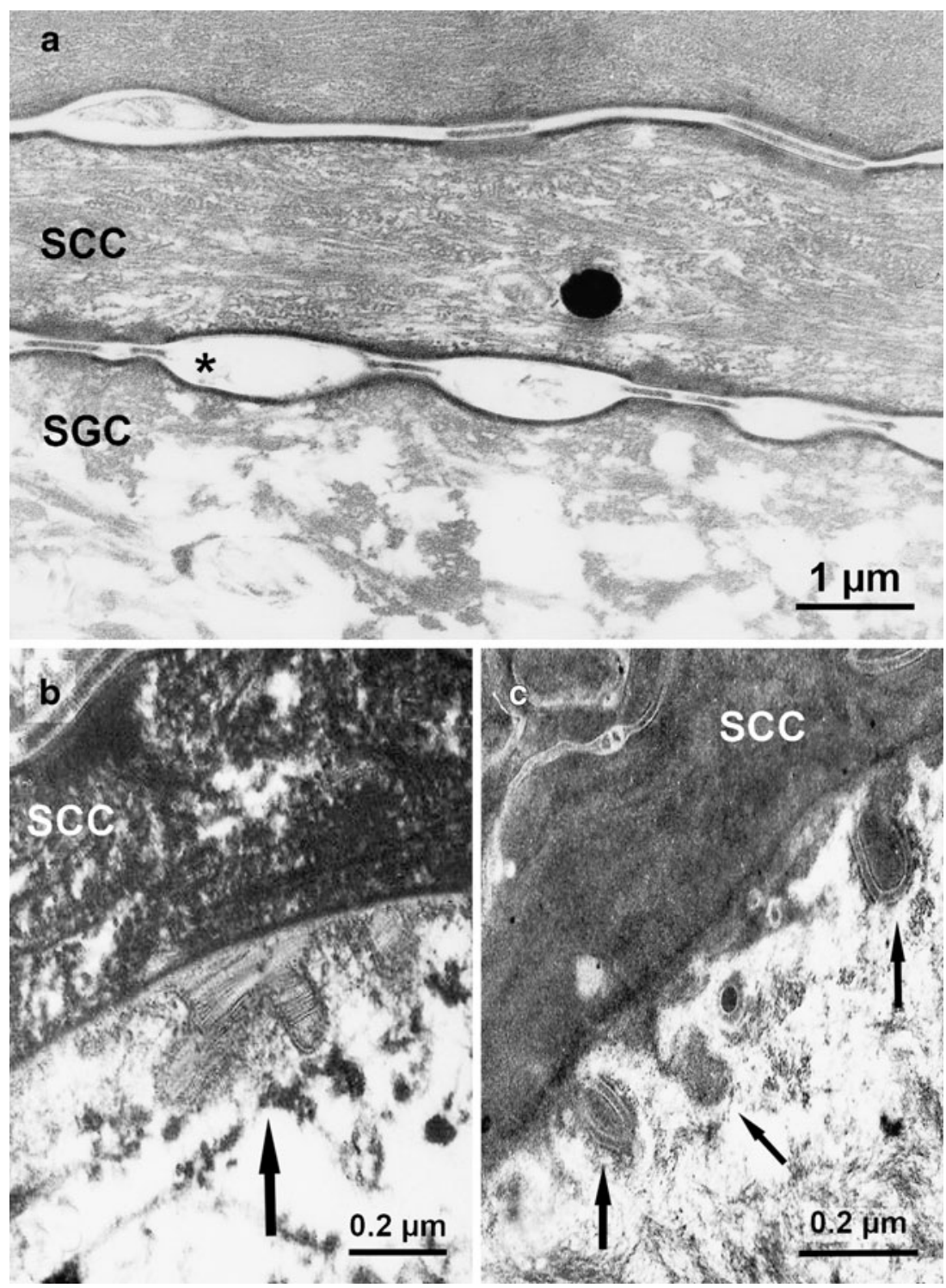

For all species, the intensity of the fluorescence staining was recorded with the help of confocal LSM and evaluated statistically regarding possible differences between the species studied (Fig. 5, diagram). As a result, it became obvious that the three species with the thickest skin (dolphin, hippopotamus and elephant) revealed significantly lower reaction intensities $(p<0.001)$ compared to all the other species, except for the African buffalo, which, however, had also a remarkable thickness $(70-80 \mu \mathrm{m})$ concerning the vital epidermis.

When the results were submitted to regression analysis, it appeared that the fluorescence intensity of the barrier region was not or only weakly correlated with any important parameter of the epidermis or hair density. In the latter case, however, the species living in the dry tropical climate showed a certain tendency of correlation between a strong reaction staining and their low hair density $(r=0.53)$.

\section{NHE-1 immunohistochemistry}

The immunohistochemical results corroborated for all wild mammals studied including those with a very thick vital epidermis, that this important antiporter and regulator of $\mathrm{pH}$ conditions in the barrier region was generally produced in the epidermis (Fig. 6a-d). Relatively strong reactions could be observed in all cells of the stratum basale and the stratum spinosum of the otter (Fig. 6a), whereas in species exhibiting rather high fluorescence intensities for glycolipids such as the Polar bear (Fig. 6b) or the Kongoni (Fig. 6c), the strongly positive reaction staining was confined to the large cells of the stratum basale. In the 
Fig. 2 Cryo-SEM results of barrier structure and exocytotic activities: a domesticated pig, the barrier (asterisk) appears as homogeneous system and two vesicles (arrows) are emptied by a cell of the stratum granulosum towards the overlying barrier into the intercellular space; b laboratory rat, numerous vesicles of the same size fuse to form or maintain the barrier zone, respectively; c domesticated pig, several small vesicles are integrated into the barrier zone. $S G C$ stratum granulosum
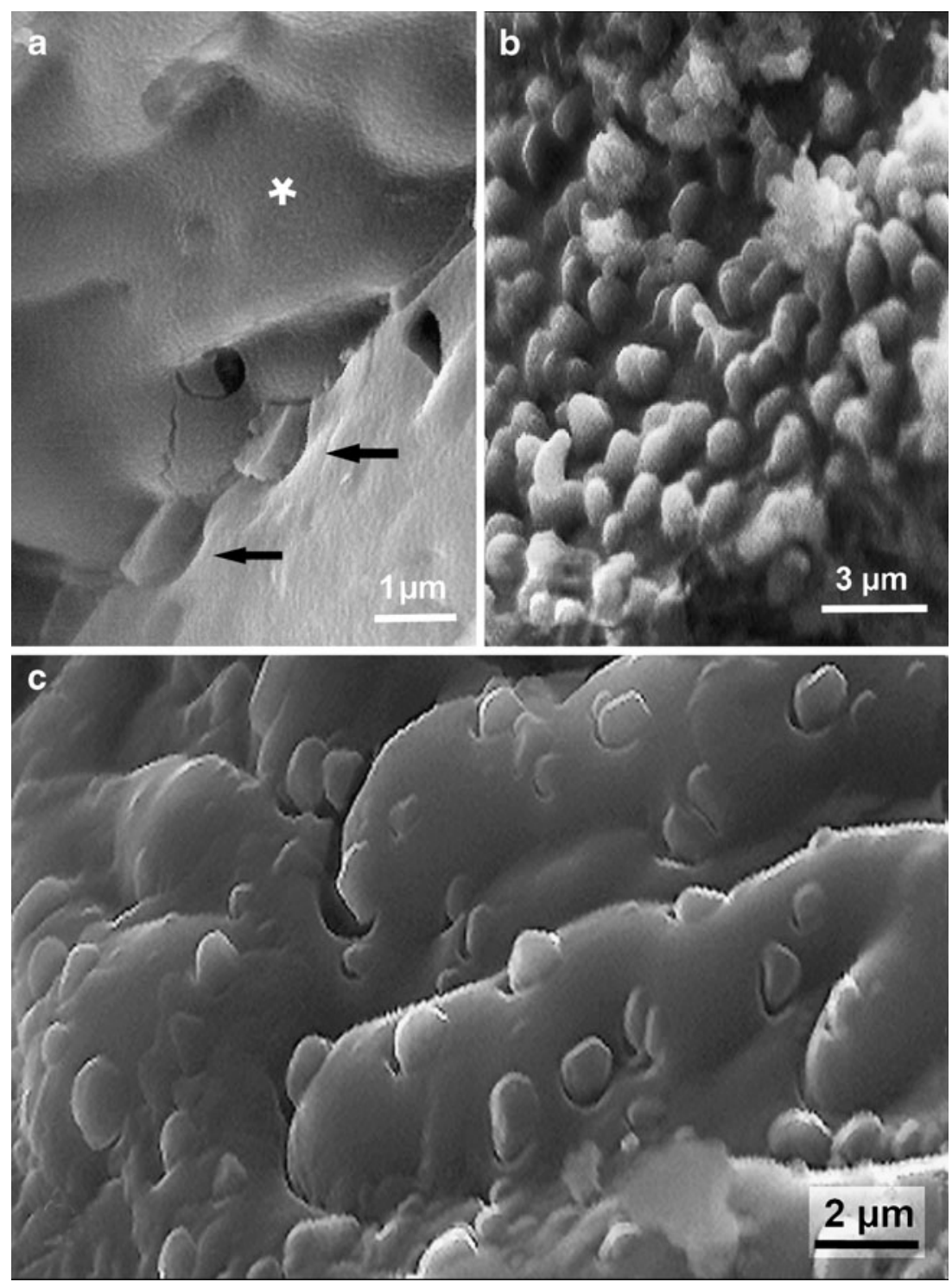

common dolphin, the hippopotamus or the African elephant, medium to strongly positive reaction fluorescence was observed in all layers of the vital epidermis, although the reactions in the stratum granulosum were somewhat weaker (Fig. 6d).

\section{Discussion}

The results obtained with a broad methodical spectrum emphasised that the epidermal barrier region in most of the wild mammals living in different habitats and climates is a clearly defined continuous border zone between the upper cells of the stratum granulosum and the inner cells of the stratum corneum conjunctum. The intercellular space between the two layers had a varying thickness between 0.1 and $3 \mu \mathrm{m}$ and was normally homogeneously filled with glycolipids from lamellar bodies, as demonstrated by TEM and our specific histochemical staining method. However, this finding did not apply to all of the species studied.

Firstly, the wild boar has to be mentioned, which displayed no specific border system but showed fine or thin glycolipid patches between the lamellae of the stratum corneum. With regard to such exception of the basic barrier structure, it is of interest that this barrier organisation is also visible in the human epidermis (e.g. Elias et al. 1977; Wertz and Downing 1982; Elias 1989; Goldsmith 1991; Jennemann et al. 2007). Therefore, we cannot generally agree with Menon and Kligman (2009), who state that the human skin barrier is unique in several ways as much due to an adaptive ability as control over the environment. Such ability seems more broadly used by other mammalian groups living in partly very different habitats! However, our findings to some extent support the use of porcine skin as model for the 
Fig. 3 Demonstration of glycolipids by HEDAF staining after embedding in water soluble plastic resin: a nutria, rather homogeneous staining of the barrier zone (arrow), but no reaction in the distinct underlying vital epidermis; b capybara, homogeneous staining of the barrier zone that shows a constant thickness; c African elephant, showing positive reactions only in the intercellular spaces of the vital epidermis; d Kudu, homogeneously stained thick barrier zone, positive reactions can also be observed in fine intercellular spaces of the vital epidermis and the basement membrane
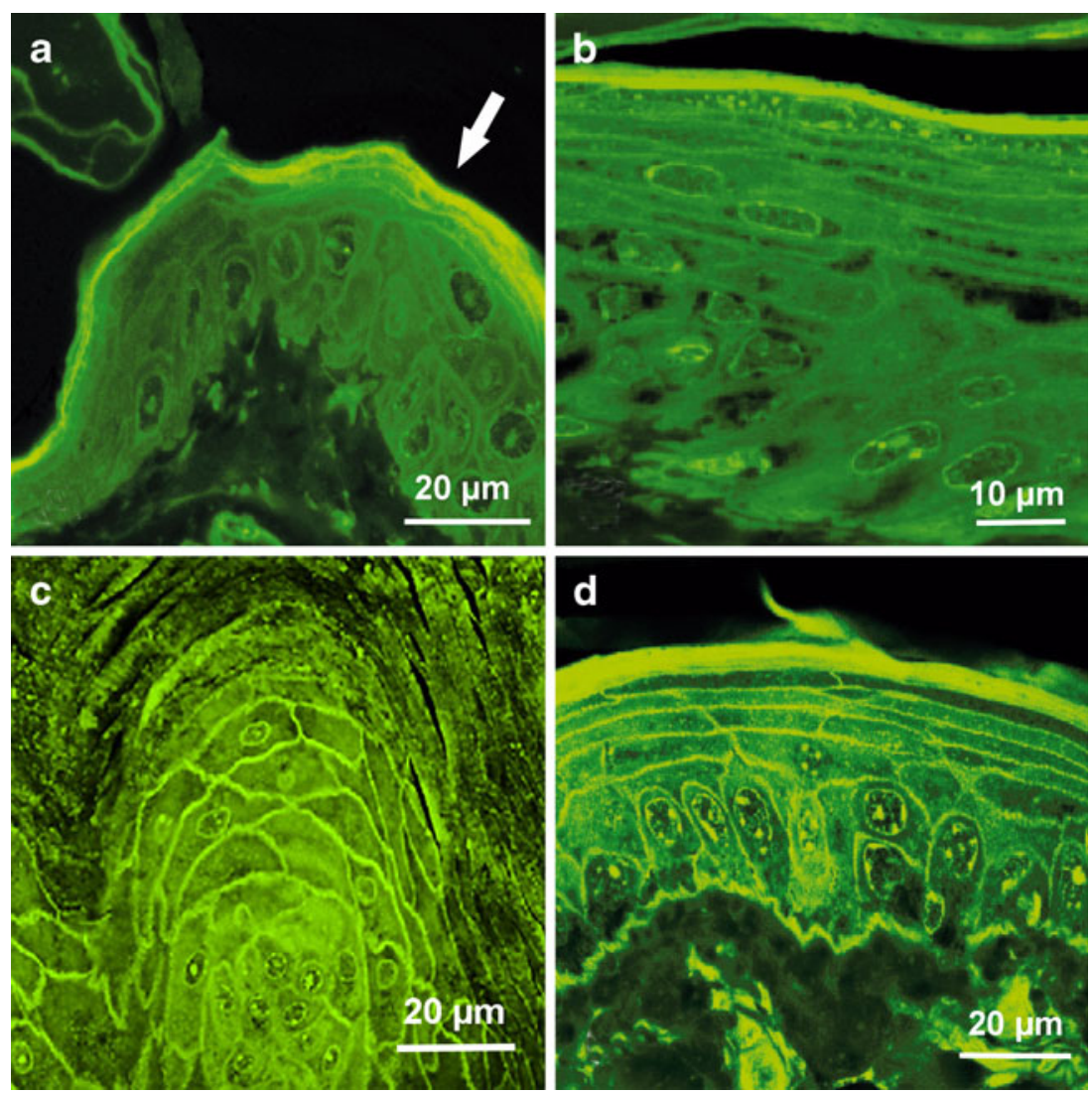

human skin (e.g. Meyer et al. 1978, 2003, 2007; Meyer 1986). Because although in the domesticated pig stratum corneum lipids are arranged predominantly in a hexagonal lattice while lipids in the human corneal layer system are predominantly packed in the denser orthorhombic lattice, porcine ceramides model human stratum corneum lipid organisation very closely (Caussin et al. 2008).

Secondly, the three large species with very thick vital epidermis have to be mentioned, the African elephant, the hippopotamus and the common dolphin. With regard to the elephant, it seems that a strong mechanical burden of the epidermis has to be balanced by a thick but not very compact stratum corneum that is produced during a rather long process. In this context, the production of glycolipids, independent of a dry and hot climate, likewise seems to proceed slowly, and our results corroborate the view of a constant disposability of glycolipid amounts in all cells of the vital epidermis as general protection against water loss. Such effect may be supported by a specific surface sculpturing of the integument in elephants, particularly in the African species, which helps to enhance retention of surface moisture, thereby impeding strong dehydration (Lillywhite and Stein 1987). In the two semiaquatic or aquatic species, respectively, the conditions against water loss are completely different, realising also that the stratum corneum is not as loosely structured as in the elephant.
Fig. 4 a Wild boar and b common seal showing positive staining reactions as confined to varying amounts of material (patches) between the corneal lamellae. $V E$ vital epidermis
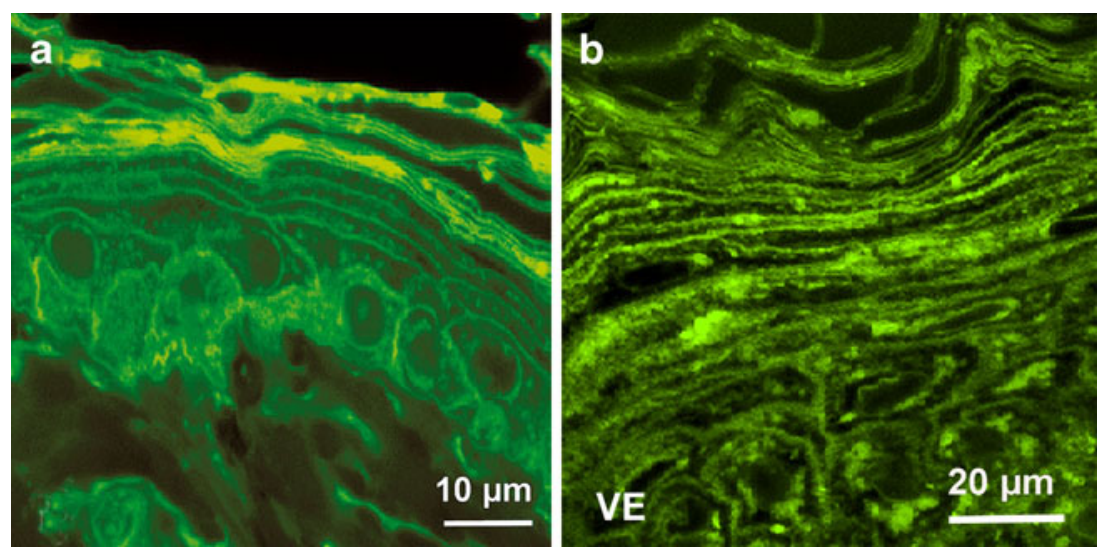
Fig. 5 Intensity of HEDAF fluorescence reaction staining in the barrier zone of the wild mammals studied; the species are grouped according to their habitats including basic climatic regions

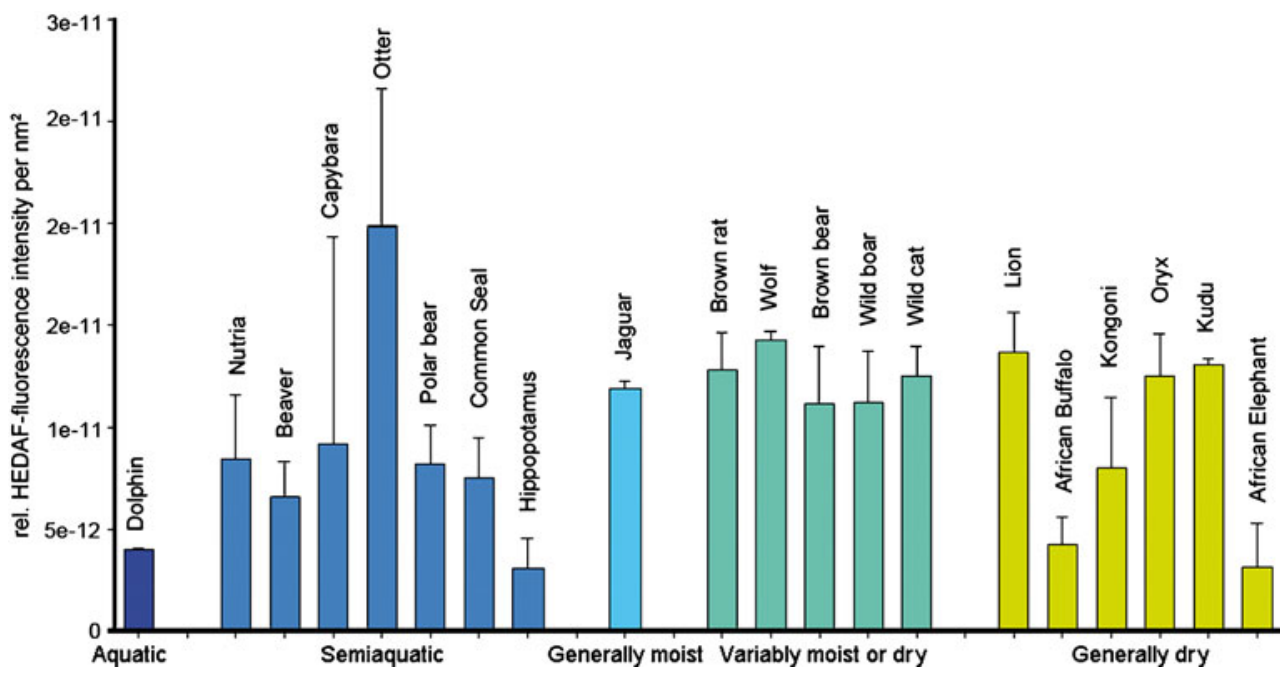

However, in both species, the amounts of glycolipids were similarily distributed as in the latter animal, whereby in the dolphin, for example, glycolipids from a multitude of fine lamellar bodies are discharged and persist in the thin intercellular spaces of the very compact stratum corneum, which, under biological biotope conditions, shows an imperceptible desquamation (Elias et al. 1987; Meyer 2010). The more relevant difference is the fact that, additionally, and unlike as in terrestrial mammals, the free fatty acids present in the keratinocytes are not transported into the intercellular spaces but accumulate increasingly in the cells of the vital epidermis, as in the dolphin and, particularly, in the hippopotamus (Menon et al. 1986; Elias et al. 1987; Pfeiffer and Jones 1993; Meyer 2010; Meyer 2007-2009, unpublished results). A change in lipid profile is possible because the lamellar bodies often contain a mixture of glycolipids and free fatty acids, as demonstrated, for example, in porcine skin (Meyer 1986; Meyer et al. 1987). The phenomenon is based on the fact that ceramides are covalently bound to cornified envelope proteins of the
Fig. 6 Immunohistochemical demonstration of the antiporter and $\mathrm{pH}$ regulator NHE-1, FITC visualisation: a otter, positive reaction staining concentrates in cells of the vital epidermis, a few cells show no reaction; b polar bear, positive reactions appear in cells of the stratum basale and stratum spinosum; c Kongoni, positive reactions in the thick epidermis are restricted to the stratum basale; d African elephant, positive reaction staining can be found in the entire vital epidermis, although more strongly in the cytoplasm of basal and spinosal cells; $S C$ stratum corneum
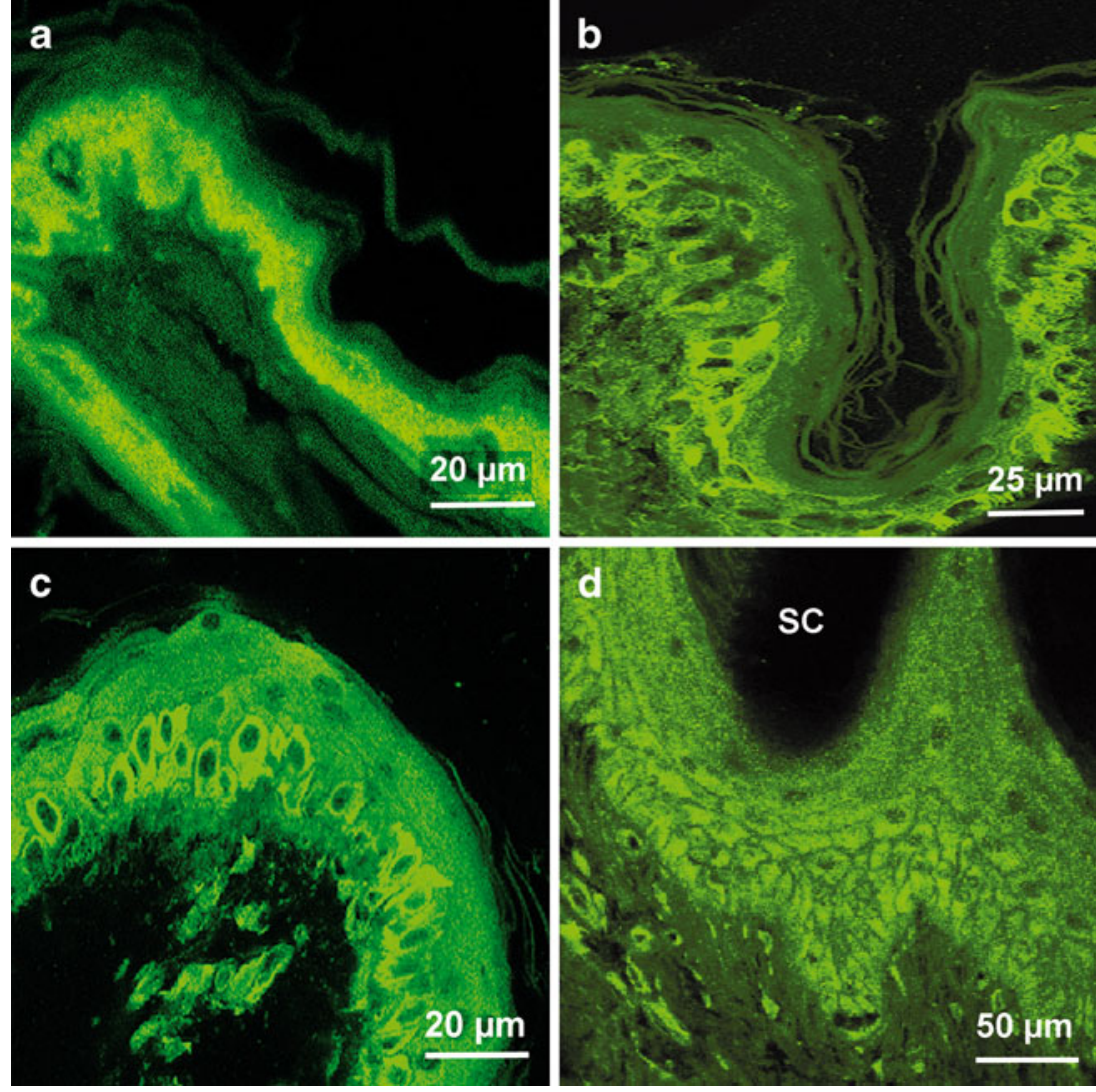
keratinocytes and form the backbone for a subsequent addition of free ceramides, free fatty acids or cholesterol (Guan et al. 2008). In summary, both large species adapted to semiaquatic or aquatic biotopes have aquired additional possibilities to compensate for the loss of a barrier layer of glycolipids.

The new technique to demonstrate glycolipids with HEDAF staining combined with embedding in a watersoluble and rather shrinkage-free plastic resin (Meyer et al. 2009) clearly helped to localise the substance more exactly within the epidermal layer system. In this way, such methodical approach generally supported the idea to get more information about any influences of the different living milieus of the wild mammals studied, by creating a better structural basis to measure the reaction intensity within the barrier layer (Fig. 5). The basic knowledge acquired confirmed that glycolipids are continuously produced to keep the important protective functions of the epidermal barrier against water loss. This information is supported by our results of a likewise continuous production of the antiporter NHE-1 that regulates the $\mathrm{pH}$ conditions for barrier formation. NHE-1 controls the acidification of extracellular microdomains that are essential for activation of $\mathrm{pH}$-sensitive enzymes to guarantee, most essentially, undisturbed repair of the epidermal permeability barrier (Behne et al. 2002; Hachem et al. 2005). We could also show, for example, that NHE-1 not only accumulates in the stratum granulosum, as demonstrated for laboratory rats (e.g. Behne et al. 2002), but also in deeper epidermal layers, particularly in species with a thick epidermis (e.g. large bovids or elephants). Such animals usually have a rather sparse hair coat that increases the danger of epidermal injuries and, thus, includes the necessity of continous barrier repair. In this way, the proliferative properties of NHE-1 are of specific importance (Slepkow et al. 2007; Hosogi et al. 2009).

Nevertheless, the most astonishing feature when evaluating the results of the glycolipid HEDAF staining statistically (without elephant, hippopotamus and dolphin, see beforehand) was the fact that relatively high staining intensities were not typically confined to the animals living in biotopes with a strong skin burden as exerted by aquatic or very dry environments. Moreover, the reaction intensity was obviously not essentially influenced by varying hair densities. However, such findings have to be discussed regarding the specific biology of most of the species studied. Primarily, the results generally corroborate the view that the body cover is perpetually endangered by several threats, such as injuries, attacks of ectoparasites and microorganisms, or strongly varying climatic influences. Furthermore, the species- or group-related qualities of the hair coat are of importance. This means that in some cases, particularly in animals with a very dense hair coat such as the otter, a better protection of epidermis integrity exists, so that during continuous glycolipid production, the substances are stored in the barrier layer, resulting in a more intense staining. In the polar bear, a constant layer of stagnant water in the underfur (Frisch et al. 1974) may activate a moderate but continuous production of barrier material. A good mechanical protection of the epidermis as generally present in species with a less dense but strong hair cover with many thick guard hairs, such as the wild boar, could slow down glycolipid production rates, as also known for epidermal keratinisation processes in these mammals (e.g. Meyer 1986; Meyer et al. 2005). On the contrary, considering the greatly varying measurement results for the capybara, for example, it has to be admitted that this animal has a very low hair coat density so that injuries and attacks of ectoparasites are not unusual (Meyer et al. 2008). Such burden includes that the production of glycolipids (and substances of innate immunity) has to be intensified very rapidly if required. This urgent matter is possible because any acute barrier disruption stimulates a repair response by activating lamellar body exocytosis with a regulation of the size and dynamics of glycosphingolipidenriched lipid raft-like domains (Feingold et al. 2007; Roelandt et al. 2009). Moreover, the demonstration of defense-related material (e.g. B-glucan receptors) present in the intercellular spaces, as shown for an endangerd epidermis like in the capybara (Meyer et al. 2008), confirms again the view of a complex barrier biology (Elias 2007). Last but not least, the skin surface temperature may activate or block epidermal barrier homeostasis by temperature sensors (members of the transient receptor potential family), in particular, when the outer temperatures distinctly increase (Denda et al. 2007) or decrease (Denda et al. 2010). This influence is of some interest for species with no hair coat or with seasonally varying hair coat qualities, but not for otters with a constantly dense fur (Kuhn 2009).

\section{Conclusions}

The epidermal barrier region of mammals living in different humid or dry habitats and climates is present as a clearly defined border region between the upper cells of the stratum granulosum and the inner cells of the stratum corneum conjunctum. Its most remarkable feature is the glycolipids forming a continuous zone (thickness 0.5 $3.5 \mu \mathrm{m})$ by the fusion of the contents of lamellar bodies, whereby a complex and rapid repair mechanism prevents an unregulated loss of water and solutes. Variations in the basic barrier zone structure had to be developed in relation to the animal size (or body size area) and hair coat density, particularly by a secondary adaptation of certain mammalian groups to the aquatic environment. In the latter case, the barrier zone system was lost, as in the hippopotamus or the 
cetaceans, but this change was successfully coupled, for example, with other basic protection aspects against water loss, such as the accumulation of free fatty acids in the epidermal cells. The fact that the skin barrier zone also stores different substances of innate immunity in the inner upwards to the outer intercellular spaces of the epidermis emphasises its important additional function as an innate immune element (e.g. Braff et al. 2005; Elias 2007).

Acknowledgements For help with skin material, we are greatly indebted to Prof. Dr. Hermann Ansorge (Goerlitz), Dr. Wolfgang Dreßen (Krefeld), Prof. Dr. Szymon Godynicki (Poznan), the late Jochen Hennig (Springe), Prof. Dr. Milan Klima (Frankfurt/M.), Prof. Dr. Wolfgang Loescher (Hannover), Prof. Dr. Andrea MeyerLindenberg (Hannover), Prof. Dr. Klaus Pohlmeyer (Hannover), the late Prof. Dr. Manfred Roehrs (Hannover), Dr. Michael Stede (Cuxhaven) and the late Prof. Dr. Helmut Wilkens (Hannover). The excellent technical assistance of Marion Gaehle and Kerstin Rohn (both University of Veterinary Medicine Hannover) is also gratefully acknowledged.

\section{References}

Aberg KM, Man MQ, Gallo RL, Ganz T, Crumrine D, Brown BE, Choi EH, Kim DK, Schroeder JM, Feingold KR, Elias PM (2008) Co-regulation and interdependence of the mammalian epidermal permeability and antimicrobial barriers. J Invest Dermatol 128:917-925

Bayliss High OB (1984) Lipid histochemistry (microscopy handbooks 6). Oxford University Press, Oxford

Behne MJ, Meyer JW, Hanson KM, Barry NP, Murata S, Crumrine D, Clegg RW, Gratton E, Holleran WM, Elias PM, Mauro TM (2002) NHE1 regulates the statum corneum permeability barrier homeostasis - microenvironment acidification assessed with fluorescence lifetime imaging. J Biol Chem 277:47399-47406

Boeck P (1989) Romeis Mikroskopische Technik, 17th edn. Urban \& Schwarzenberg, Muenchen

Bollhorn M (1999) Histologische und histochemische Untersuchungen am Integument des Seehunds (Phoca vitulina). Dissertation thesis, University of Veterinary Medicine Hannover, Hannover, Germany

Braff MH, Di Nardo A, Gallo RL (2005) Keratinocytes store the antimicrobial peptide cathelicidin in lamellar bodies. J Invest Dermatol 124:394-400

Caussin J, Gooris GS, Janssens M, Bouwstra JA (2008) Lipid organization in human and porcine stratum corneum differs widely, while lipid mixtures with porcine ceramides model human stratum corneum lipid organization very closely. Biochim Biophys Acta Biomembr 1778:1472-1482

Delacour D, Koch A, Ackermann W, Eude-Le Parco I, Elsaesser HP, Poirier F, Jacob R (2008) Loss of galectin-3 impairs membrane polarisation of mouse enterocytes in vivo. J Cell Sci 121:458-465

Denda M, Sokabe T, Fukumi-Tominaga T, Tominaga M (2007) Effects of skin surface temperature on epidermal permeability barrier homeostasis. J Invest Dermatol 127:654-659

Denda M, Tsutsumi M, Denda S (2010) Topical application of TRPM8 agonists accelerates skin permeability barrier recovery and reduces epidermal proliferation induced by barrier insult: role of cold-sensitive TRP receptors in epidermal permeability barrier homoeostasis. Exp Dermatol 19:791-795

Elias PM (1989) The stratum corneum as an organ of protection: old and new concepts. Curr Probl Dermatol 18:10-21
Elias PM (2007) The skin barrier as an innate immune element. Sem Immunopathol 29:3-14

Elias PM, Goerke J, Friend DS (1977) Mammalian epidermal barrier layer lipids: composition and influence on structure. J Invest Dermatol 69:535-546

Elias PM, Menon GK, Grayson S, Brown BE, Rehfeld SJ (1987) Avian sebokeratocytes and marine mammal lipokeratinocytes: structural, lipid biochemical, and functional considerations. Am J Anat 180:161-177

Feingold KR, Schmudt M, Elias PM (2007) The regulation of permeability barrier homeostasis. J Invest Dermatol 127:1574-1576

Frisch J, Øritsland NA, Krog J (1974) Insulation of furs in water. Comp Biochem Physiol 47:403-410

Gerrits PO, Smid L (1983) A new, less toxic polymerisation system for the embedding of soft tissues in glycol methacrylate and subsequent preparing of serial sections. J Microsc 132:81-85

Goldsmith LA (ed) (1991) Physiology, biochemistry, and molecular biology of the skin, 2nd edn. Oxford University Press, New York

Guan GP, Zhou MN, Xu AE, Kang KF, Liu JF, Wei XD, Li YW, Zhao DK, Hong WS (2008) The skin: an indispensable barrier. Exp Dermatol 17:1059-1062

Hachem JP, Crumrine D, Fluhr J, Brown BE, Feingold KR, Elias PM (2003) $\mathrm{pH}$ directly regulates epidermal permeability barrier homeostasis, and stratum corneum integrity/cohesion. J Invest Dermatol 121:345-353

Hachem JP, Behne M, Aronchik I, Demerjian M, Feingold KR, Elias PM, Mauro TM (2005) Extracellular pH controls NHE1 expression in epidermis and keratinocytes: implications for barrier repair. J Invest Dermatol 125:790-797

Hamanaka S, Nakazawa S, Yamanaka M, Uchida Y, Otsuka F (2005) Glucosylceramide accumulates preferentially in lamellar bodies in differentiated keratinocytes. Br J Dermatol 152:426-434

Hanstede JG, Gerrits PO (1983) The effects of embedding in watersoluble plastics on the final dimensions of liver sections. J Microsc 131:79-86

Hautzer NW, Wittkuhn JF, McCaughey WTE (1980) Trypsin digestion in immunoperoxidase staining. J Histochem Cytochem 28:52-53

Heung LJ, Luberto C, Del Poeta M (2006) Role of sphingolipids in microbial pathogenesis. Infect Immun 74:28-39

Holleran WM, Tagaki Y, Menon GK, Legler G, Feingold KR, Elias PM (1993) Processing of epidermal glucosylceramide is required for optimal mammalian cutaneous permeability function. J Clin Invest 91:1656-1664

Holleran WM, Tagaki Y, Uchida Y (2006) Epidermal sphingolipids: metabolism, function, and roles in skin disorders. FEBS Lett 580:5456-5466

Hornickel I, Kacza J, Schnapper A, Beyerbach M, Schoennagel B, Seeger J, Meyer W (2011) Demonstration of substances of innate immunity in the esophagus epithelium of domesticated mammals. Part I-methods and comparative fixation evaluation. Acta Histochem (in press). doi:10.1016/j.acthis.2009.09.009

Hosogi S, Takemura Y, Miyazaki H, Niisato N, Iwasaki Y, Marunaka Y (2009) Sodium hydrogen exchanger isoform 1 (NHE-1)dependent proliferation of gastric cancer cells. J Physiol Sci 59 (Suppl 1):303

Jennemann R, Sandhoff R, Langbein L, Kaden S, Rothermel U, Gallala H, Sandhoff K, Wiegandt H, Grone HJ (2007) Integrity and barrier function of the epidermis critically depend on glucosylceramide synthesis. J Biol Chem 282:3083-3094

Karnovsky MJ (1965) A formaldehyde-glutaraldehyde fixative of high osmolality for use in electron microscopy. J Cell Biol 27:137A$138 \mathrm{~A}$

Koliakos G, Paletas K, Kaloyianni M (2008) NHE-1: a molecular target for signalling and cell matrix interactions. Connect Tissue Res 49:157-161 
Kuhn RA (2009) Comparative analysis of structural and functional hair coat characteristics, including heat loss regulation, in the Lutrinae (Carnivora: Mustelidae). Ph.D. thesis, University of Hamburg, Hamburg, Germany

Lillie RD, Fullmer HM (1976) Histopathologic technic and practical histochemistry. McGraw-Hill, New York

Lillywhite HB (2006) Water relations of tetrapod integument. J Exp Biol 209:202-226

Lillywhite HB, Stein BR (1987) Surface sculpturing and water retention of elephant skin. J Zool 211:727-734

Luft JH (1961) Improvements in epoxy resin embedding methods. J Biophys Biochem Cytol 9:409-414

Menon GK, Kligman AM (2009) Barrier functions of human skin: a holistic view. Skin Pharmacol Physiol 22:178-189

Menon G, Grayson S, Brown BE, Elias PM (1986) Lipokeratinocytes of the epidermis of a cetaceen (Phocena phocena). Histochemistry, ultrastructure, and lipid composition. Cell Tissue Res 244:385-394

Meyer W (1986) Die Haut des Schweines (The porcine integument). Schlüter, Hannover

Meyer W (2010) The integument of dolphins (Animal science, issues and professions series). Nova Science, New York

Meyer W, Hornickel I (2011) Tissue fixation - the most underestimated methodical feature of immunohistochemistry. In: Méndez-Vilas A, Diaz J (eds) Microscopy: science, technology, applications and education (microscopy book series, vol. 4). Formatex Research Center, Badajoz (in press)

Meyer W, Schwarz R, Neurand K (1978) The skin of domestic mammals as a model for the human skin, with special reference to the domestic pig. Curr Probl Dermatol 7:39-52

Meyer W, Uhr G, Schwarz R (1981) Untersuchungen an der Haut der Europäischen Wildkatze (Felis silvestris Schreber). I. Hautschichten und Hautdrüsen. Zool Jb Anat 106:163-185

Meyer W, Schlesinger C, Neurand K (1987) Membrane-coating granules (MCGs) in porcine epidermis. Schweiz Arch Tierheilkde 129:133-137

Meyer W, Zschemisch NH, Godynicki S (2003) The porcine ear skin as a model system for the human integument: Influence of storage conditions on basic features of epidermis structure and function - a histological and histochemical approach. Pol J Vet Sci 6:17-28

Meyer W, Zschemisch NH, Lehmann H, Busche R, Kunz U (2005) Comparative distribution of sulphur, thiols and disulphides in the porcine stratum corneum. Eur J Dermatol 15:243-250
Meyer W, Kacza J, Zschemisch NH, Godynicki S, Seeger J (2007) Observations on the actual structural conditions in the stratum superficiale dermidis of porcine ear skin, with special reference to its use as model for human skin. Ann Anat 189:143-156

Meyer W, Kloepper JE, Fleischer LG (2008) Demonstration of Bglucan receptors in the skin of aquatic mammals - a preliminary report. Eur J Wildl Res 54:479-486

Meyer W, Schmidt J, Busche R, Jacob R, Naim HY (2009) Demonstration of lipids in plastic resin-embedded sections of skin material. J Microsc 233:5-9

Millonig G (1961) Advantages of a phosphate buffer for $\mathrm{OsO}_{4}$ solutions in fixation. J Appl Phys 32:1637

Mizutani Y, Mitsutake S, Tsuji K, Kihara A, Igarashi Y (2009) Ceramide biosynthesis in keratinocyte and its role in skin function. Biochimie 91:784-790

Pearse AGE (1985) Histochemistry. Theoretical and applied, 4th edn, vol. 2: analytical technology. Churchill Livingstone, Edinburgh

Pfeiffer CJ, Jones FM (1993) Epidermal lipid in several cetacean species: ultrastructural observations. Anat Embryol 188:209-218

Reynolds ES (1963) The use of lead citrate of high $\mathrm{pH}$ as an electron opaque stain in electron microscopy. J Cell Biol 17:208-212

Richardson KC, Jarett L, Finke EH (1960) Embedding in epoxy resins for ultrathin sectioning in electron microscopy. Stain Technol 35:313-323

Roelandt T, Giddelo C, Heughebaert C, Denecker G, Hupe M, Crumrine D, Kusuma A, Haftek M, Roseeuw D, Declercq W, Feingold KR, Elias PM, Hachem JP (2009) The 'caveolae brake hypothesis' and the epidermal barrier. J Invest Dermatol 129:927-936

Slepkow ER, Rainey JK, Sykes BD, Fliegel L (2007) Structural and functional analysis of $\mathrm{Na}+\mathrm{H}+$ exchanger. Biochem J 401:623-633

Stempak JG, Ward RT (1964) An improved staining method for electron microscopy. J Cell Biol 22:697-701

Uhr G (1984) Vergleichende Untersuchungen an Haut und Haarkleid von Mus musculus domesticus Rutty, 1772 und Rattus norvegicus Berkenhout, 1769. Diss rer nat thesis, University of Hannover, Hannover, Germany

Wenneckes T, van den Berg RJBH, Boot RG, van der Marel GA, Aerts JMFG (2009) Glycosphingolipids-nature, function, and pharmacological modulation. Angew Chem Int Ed 48:8848-8869

Wertz PW, Downing DT (1982) Glycolipids in mammalian epidermis: structure and function in the water barrier. Science 217:12611262 